\title{
Multiloculated Hydrocephalus : Open Craniotomy or Endoscopy?
}

\author{
Yun Ho Lee, M.D., ${ }^{1}$ Young Sub Kwon, M.D., ${ }^{1,2}$ Kook Hee Yang, M.D., Ph.D. \\ Department of Neurosurgery, National Health Insurance Service Ilsan Hospital, Goyang, Korea \\ Department of Neurosurgery, ${ }^{2}$ School of Medicine, Kangwon National University, Chuncheon, Korea
}

Multiloculated hydrocephalus (MLH) is a condition in which patients have multiple, separate abnormal cerebrospinal fluid collections with no communication between them. Despite technical advancements in pediatric neurosurgery, neurological outcomes are poor in these patients and the approach to this pathology remains problematic especially given individual anatomic complexity and cerebrospinal fluid (CSF) hydrodynamics. A uniform surgical strategy has not yet been developed. Current treatment options for MLH are microsurgical fenestration of separate compartments by open craniotomy or endoscopy, shunt surgery in which multiple catheters are placed in the compartments, and combinations of these modalities. Craniotomy for fenestration allows better visualization of the compartments and membranes, and it can offer easy fenestration or excision of membranes and wide communication of cystic compartments. Hemostasis is more easily achieved. However, because of profound loss of CSF during surgery, open craniotomy is associated with an increased chance of subdural hygroma and/or hematoma collection and shunt malfunction. Endoscopy has advantages such as minimal invasiveness, avoidance of brain retraction, less blood loss, faster operation time, and shorter hospital stay. Disadvantages are also similar to those of open craniotomy. Intraoperative bleeding can usually be easily managed by irrigation or coagulation. However, handling of significant intraoperative bleeding is not as easy. Currently, endoscopic fenestration tends to be performed more often as initial treatment and open craniotomy may be useful in patients requiring repeated endoscopic procedures.

Key Words : Craniotomy $\cdot$ Endoscopy $\cdot$ Hydrocephalus $\cdot$ Multiloculated.

\section{INTRODUCTION}

Multiloculated hydrocephalus (MLH) is a condition in which patients have multiple, separated abnormal cerebrospinal fluid (CSF) collections with no communication between them. Uniloculated hydrocephalus is defined as cystic dilatation of one part of the normal ventricular system such as obstruction of one of the foramina of Monroe or an isolated fourth ventricle obstruction $^{3)}$. MLH is also referred to as multilocular hydrocephalus, multiseptate hydrocephalus, polycystic hydrocephalus, ventricular compartmentalization, intraventricular septations and multicompartmental hydrocephalus ${ }^{1,26)}$.

Etiologies of MLH include complications of neonatal meningitis and germinal matrix hemorrhage in premature neonates. Other causes include post-shunt infection, overdrainage in shunted hydrocephalus, direct parenchymal injury inflicted

- Received : January 23, 2017 •Revised : March 21, 2017 •Accepted : March 27, 2017

- Address for reprints : Kook Hee Yang, M.D., Ph.D.

Department of Neurosurgery, National Health Insurance Service Ilsan Hospital, 100 Ilsan-ro, Ilsandong-gu, Goyang 10444, Korea

Tel : +82-31-900-0248, Fax: +82-31-900-0588, E-mail : khyang@nhimc.or.kr

This is an Open Access article distributed under the terms of the Creative Commons Attribution Non-Commercial License (http://creativecommons.org/licenses/by-nc/4.0) which permits unrestricted non-commercial use, distribution, and reproduction in any medium, provided the original work is properly cited. 
by a catheter tip, head trauma, and intracranial surgery ${ }^{2,5,7,10,14)}$. Predisposing factors include low birth weight, prematurity, perinatal complications, and congenital malformation ${ }^{26)}$. Although the full pathogenesis of MLH remains unclear, it is known that inflammation leads to subependymal gliosis, which produces glial tufts and septations that occlude the normal ventricle system. Septations also develop via organization of intraventricular exudate and debris from ventriculitis $^{9,22-24)}$.

Despite technical advancements in pediatric neurosurgery, neurological outcomes are poor in these patients and the approach to this pathology remains problematic. The mortality rate has decreased as a result of improved neonatal care; however, psychomotor retardation is a very serious problem in these patients. About $90 \%$ of these patients have severe cognitive deficits and require assistance with activities of daily living $^{6,26)}$.

Patients with MLH have individual anatomic complexity and CSF hydrodynamics. Therefore strategies for management of MLH are diverse and creativity is often required. Multimodal surgical options exist for the treatment of MLH, including the insertion of multiple shunts into each dilated CSF cavity, fenestration of septate membranes by open craniotomy and/or endoscopy, and combined approaches. However, the optimal treatment strategy has not yet been identified. In this review we will discuss surgical options for MLH, and the pros and cons of each surgical option will be discussed based on previously published reports.

\section{HISTORY OF SURGICAL MODALITIES}

Currently, treatment options for MLH are microsurgical fenestration of septate compartments by open craniotomy or endoscopy, shunt surgery in which multiple catheters are placed in multilobulated compartments, and a combination of these modalities ${ }^{24)}$. However, considering the complexity of the disease and the fact that a majority of patients are neonates and infants, effective management of progressive MLH is very challenging.

Generally, a single shunt is not useful in MLH and a multimodal approach is needed. In the past the traditional surgical treatment of MLH was implantation of a multiple shunt system in each CSF-filled space. However, there are several dis- advantages to the multiple shunt system. First of all, multiple catheters may not adequately drain all CSF-filled cavities. Partial or complete malfunction of each catheter system can lead to aggravation of other lobulated areas. In such cases it is difficult to identify which catheter in the shunt system is problematic, making management quite complicated. The risk of shunt infection also increases as the number of operative procedures and amount of implanted hardware increases. It is therefore preferable to avoid multiple shunt catheters if possible ${ }^{4,16,22)}$.

Fenestration by open craniotomy was first described by Rhoton and Gomez ${ }^{20)}$ in 1972. A ten-week-old baby with rapid head growth was diagnosed with progressive multi-septate hydrocephalus due to previous E. coli meningitis. The hydrocephalus was controlled with a single shunt placed via a small craniotomy and transcallosal approach after direct wide excision of multi-septated membranes through a small cortical incision.

The role of endoscopic fenestration for the treatment of compartmentalized hydrocephalus was first described by Kleinhaus et al. ${ }^{11)}$ in 1982. Endoscopic fenestration was performed in a patient with post-infectious hydrocephalus who required bilateral shunt catheters, and communication was established between the compartmentalized cyst and the ventricular system prior to placement of the indwelling shunt system. Endoscopic surgery has recently emerged as an option in the treatment of MLH, but these techniques were previously used in only limited situations because of the high mortality and morbidity. However, since Lewis et al. ${ }^{13)}$ first published a large series of patients who underwent endoscopic surgery for loculated hydrocephalus in 1995, endoscopic procedures are now used frequently for treatment of MLH worldwide.

Although fenestration of all compartments by open craniotomy or endoscopy is successful, a shunt is almost always required. Thus the goal of open microsurgery or endoscopy is to make multiple compartments into a single CSF compartment to allow for drainage via a single shunt catheter system (Fig. 1). So far, the best choice of treatment for MLH has not been determined. In the treatment of patients with MLH, the most important goals are to relieve intracranial hypertension and decrease morbidity by minimizing the number of operations and complications associated with multiple shunt catheters regardless of what methods are used. 

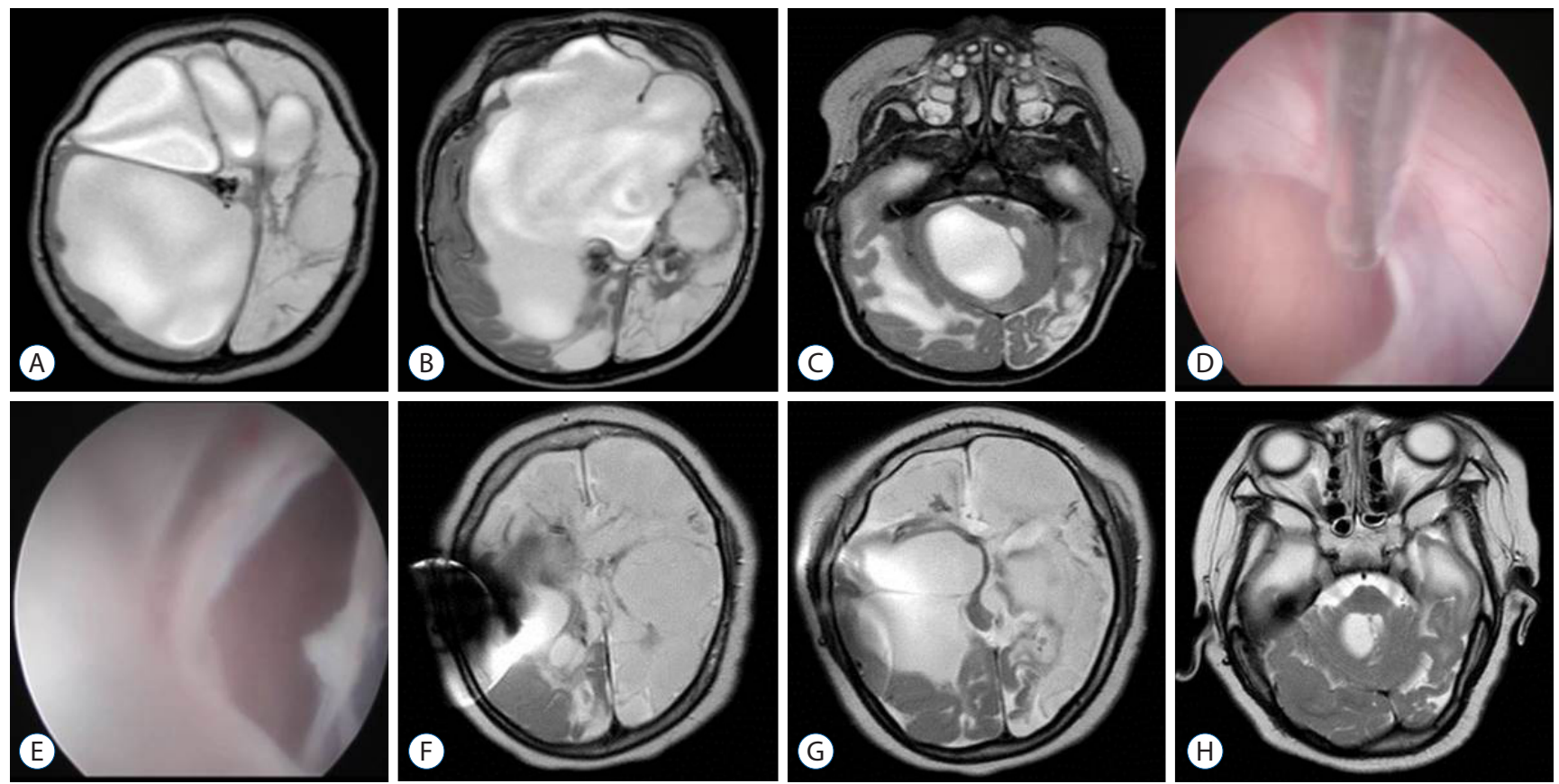

Fig. 1. A six-month-old female was diagnosed with multiloculated hydrocephalus on MRI due to previous neonatal meningitis. A-C : Preoperative axial T2 images showed multiloculated hydrocephalus with severe cerebromalacia; D and E : Photographs of endoscopic fenestration. F-H : Follow up MRI after two years showed the ventricles had decreased in size and demonstrated one proximal indwelling shunt catheter via the right Kocher's point. During the follow up period, the patient did not require revision of the shunt. MRI : magnetic resonance imaging.

\section{OPEN CRANIOTOMY FOR FENESTRATION OF MLH}

Since the suggestion of open craniotomy by Rhoton and Gomez in 1972, this surgical approach has increased in popularity. Nida and colleagues ${ }^{15)}$ reported that ten patients with MLH were treated with open craniotomy. They performed transcallosal fenestration of intraventricular septation in six patients and multiple shunt procedures in the remaining four patients. In the open craniotomy group, the shunt revision rate decreased from a median 2.75 cases per year prior to fenestration to 0.25 cases per year following fenestration. This is in comparison to a median shunt revision rate of 0.55 cases per year in the group of patients who underwent the shunt procedure only without fenestration. However, the transcallosal approach has several risks such as venous infarction resulting from sacrifice of bridging veins during retraction of the brain, disconnection syndromes due to the incised corpus callosum, and damage to the fornix leading to memory impairment ${ }^{13)}$. Another study reported surgical fenestration through open craniotomy in 33 cases of MLH. The authors also used endoscopic fenestration in relatively simple pathologies such as uniloculated hydrocephalus, while they preferred open craniotomy for fenestration of MLH. Open craniotomy was performed via a posterior parietal approach and cortisectomy was made closest to the loculated ventricle for fenestration. Nineteen of 33 patients needed additional fenestration and the remaining 14 patients did not require additional surgery during a median 3.7 years of follow-up. In 25 of 33 patients $(76 \%)$, only one ventricular catheter was required, two catheters in six and three catheters in one patient. One patient did not require a shunt system ${ }^{22)}$.

\section{Advantages and disadvantages of open cranioto- my over endoscopy}

Craniotomy for fenestration has several advantages. First of all, it allows better visualization of the compartments and membranes. Therefore, it enables easy fenestration or excision of membranes, and wide communication of the cystic compartments, which allows the possibility of avoiding placement of multiple shunt catheters. Additionally, hemostasis is more easily achieved with conventional microscopic techniques ${ }^{22)}$. However, patients who undergo open craniotomy more frequently develop subdural hygroma and/or hematoma because of the profound loss of CSF during surgery. Loss of CSF can 
lead to ventricular collapse and sagging of the brain, and the chances of shunt malfunction also increase. There is more blood loss in open craniotomy, which can increase surgical morbidity in neonates and infants ${ }^{133}$.

\section{ENDOSCOPY FOR FENESTRATION OF MLH}

Since the study of endoscopic fenestration for intraventricular cysts by Kleinhaus and colleagues in 1982, there were several reports detailing the endoscopic approach for MLH until the mid $1990 \mathrm{~s}^{12,17,18)}$. However, most were case reports, and long-term results were unknown. In 1995 Lewis and colleagues ${ }^{13)}$ reported the first large series of patients who underwent endoscopic treatment (34 cases in total: $13 \mathrm{MLH}$ and 21 uniloculated hydrocephalus). Six (46\%) of the 13 patients with MLH required one or more additional endoscopic procedures. The shunt revision rate decreased from 3.04 cases per year to 0.25 cases after endoscopic fenestration for patients with MLH. Therefore, the authors proposed that endoscopic fenestration was a safe and less invasive technique as initial treatment for MLH. Nowos $\Varangle$ awska and associates ${ }^{16)}$ reported that there was a significant reduction in shunt revision rate and complications, especially shunt infection, after endoscopic treatment (47 patients) compared to conventional shunt operations (80 patients) in patients with complex compartmentalized hydrocephalus. Only thirteen of 80 patients who underwent conventional shunt techniques were successfully treated with implantation of a simple shunt system, whereas the shunt system was able to be simplified in 30 of the 47 endoscopically-treated patients. In addition, patients treated with endoscopy had better postoperative clinical status than the other group. Several other studies compared the outcome of endoscopic fenestration with multiple shunt catheters (shunt surgery only), and concluded that the use of endoscopic fenestration can reduce revision rates compared to shunt surgery alone. However, the majority of these reports did not discriminate between uniloculated hydrocephalus and MLH ${ }^{3,4,6,13,26)}$.

One study compared open craniotomy with endoscopic fenestration for the treatment of MLH. Twelve of a total of 25 patients underwent open craniotomy and the remaining 13 patients underwent endoscopic fenestration. Shunt-related procedures and shunt revision rate were not significantly different between the two groups. There was an average of 5.1 \pm
4.5 cases of subsequent shunt-related interventions per year in the open craniotomy group versus $3.1 \pm 4.0$ cases per year in the endoscopy group ( $p$-value $=0.25$ ). The median shunt revision rate for the open craniotomy group was 0.74 revisions per year versus 0.5 revisions per year in the endoscopy group ( $p$-val$\mathrm{ue}=0.51$ ). Advantages of endoscopic fenestration included decreased blood loss, shorter operative time, and shorter hospital stay. However, there were some limitations to that study including the fact that there were several surgeons, it was a retrospective analysis and there was bias associated with procedure selection according to disease severity. Furthermore, predetermined specific clinical and radiological criteria were not considered when selecting surgical options ${ }^{1)}$.

\section{Advantages and disadvantages of endoscopy over open craniotomy}

In the past decade endoscopic procedures have been developed to minimize the invasiveness of conventional microneurosurgery. As endoscopy techniques and devices have advanced, the use of endoscopy has become more frequent than open craniotomy in the treatment of MLH.

Using the endoscope has advantages such as less invasiveness, avoidance of brain retraction, less blood loss, faster operation time, and shorter hospital stay. It also has several disadvantages. A subdural hematoma and/or hygroma may form, but the incidence of this is lower than with open craniotomy. The risk of ventriculitis, CSF leakage (subcutaneous CSF collection), and hemorrhage are reported to be similar to that with open craniotomy. Intraoperative bleeding can usually be easily managed by irrigation or coagulation. However, handling of significant intraoperative bleeding is not as easy in endoscopy as in open craniotomy ${ }^{22,24,25)}$.

\section{Role of navigation in endoscopy}

Since Rhoten and coworkers ${ }^{19)}$ first described the use of neuronavigation with endoscopy during intracranial procedures, several frameless stereotactic systems (neuronavigation) have been used in neurosurgery in recent years. The patient anatomy is distorted in MLH due to previous infection, hemorrhage, congenital intracranial anomaly, which may lead to difficulties with orientation during endoscopy and/or open craniotomy. Neuronavigation is therefore helpful in this setting. Besides facilitating the identification of complex anatomy throughout the procedure, the entry point and fenestration 
trajectory can be planned preoperatively through preoperative simulation $^{8,21)}$. Because the majority of these patients are young children, neonates and infants, electromagnetic-guided neuronavigation is used frequently where rigid fixation is not required and changes in head position are possible during surgery $^{8)}$. Brain shift is a frequently encountered problem in neuronavigation. However, if the cysts are not large, there may be fewer shifts during endoscopy than in open craniotomy. Continuous intraoperative irrigation also leads to less collapse of the ventricle and therefore less brain shift. Ultrasound can be helpful in some cases. Intraoperative magnetic resonance images can also be used, but this is not cost effective.

\section{CONCLUSION}

Because the disease is very complex and patient conditions vary significantly in MLH, it is difficult to compare the usefulness of endoscopy with open craniotomy as initial surgical management. Currently, endoscopic fenestration tends to be performed more often as initial treatment and open craniotomy may be useful in patients requiring repeat endoscopic procedures. However, this is not because endoscopic procedures are superior, but because of their minimally invasive nature and decreased surgical morbidity and mortality. Therefore, surgical options should be carefully considered depending on the individual case.

\section{References}

1. Akbari SHA, Holekamp TF, Murphy TM, Mercer D, Leonard JR, Smyth MD, et al. : Surgical management of complex multiloculated hydrocephalus in infants and children. Childs Nerv Syst 31 : 243-249, 2015

2. Albanese V, Tomasello F, Sampaolo $S$ : Multiloculated hydrocephalus in infants. Neurosurgery $8: 641-646,1981$

3. Andresen $M$, Juhler $M:$ Multiloculated hydrocephalus: a review of current problems in classification and treatment. Childs Nerv Syst 28 : 357-362, 2012

4. Cipri S, Gambardella G : Neuroendoscopic approach to complex hydrocephalus. Personal experience and preliminary report. J Neurosurg Sci $45:$ : 92-96, 2001

5. Eder HG, Leber KA, Gruber W : Complications after shunting isolated IV ventricles. Childs Nerv Syst 13 : 13-16, 1997

6. El-Ghandour NM : Endoscopic cyst fenestration in the treatment of multiloculated hydrocephalus in children. J Neurosurg Pediatr 11 : 402-409,
2013

7. Eller TW, Pasternak JF : Isolated ventricles following intraventricular hemorrhage. J Neurosurg 62 : 357-362, 1985

8. Hermann EJ, Esmaeilzadeh M, Ertl P, Polemikos M, Raab P, Krauss JK : Endoscopic intracranial surgery enhanced by electromagnetic-guided neuronavigation in children. Childs Nerv Syst 31 : 1327-1333, 2015

9. Jamjoom A, Mohammed A, Al-Boukai A, Jamjoom Z, Rahman N, Jamjoom $\mathrm{H}$ : Multiloculated hydrocephalus related to cerebrospinal fluid shunt infection. Acta Neurochir $138:$ 714-719, 1996

10. Kalsbeck JE, DeSousa AL, Kleiman MB, Goodman JM, Franken EA : Compartmentalization of the cerebral ventricles as a sequela of neonatal meningitis. J Neurosurg 52 : 547-552, 1980

11. Kleinhaus S, Germann R, Sheran M, Shapiro K, Boley SJ : A role for endoscopy in the placement of ventriculoperitoneal shunts. Surg Neurol 18 : 179-180, 1982

12. Lewis Al, Crone KR : Advances in neuroendoscopy. Contemp Neurosurg $16: 1-6,1994$

13. Lewis Al, Keiper GL Jr, Crone KR : Endoscopic treatment of loculated hydrocephalus. J Neurosurg 82 : 780-785, 1995

14. Marquardt G, Setzer M, Lang J, Seifert V : Delayed hydrocephalus after resection of supratentorial malignant gliomas. Acta Neurochir (Wien) 144 : 227-231; discussion 231, 2002

15. Nida TY, Haines SJ : Multiloculated hydrocephalus: craniotomy and fenestration of intraventricular septations. J Neurosurg 78 : 70-76, 1993

16. Nowos ławska E, Polis L, Kaniewska D, Miko łajczyk W, Krawczyk J, Szymański $W$, et al. : Effectiveness of neuroendoscopic procedures in the treatment of complex compartmentalized hydrocephalus in children. Childs Nerv Syst 19 : 659-665, 2003

17. Powers $S$ : Fenestration of intraventricular cysts using a flexible, steerable endoscope in Bauer BL, Hellwig D (eds) : Minimally Invasive Neurosurgery, ed 1. Vienna : Springer, 1992, Vol 54, pp42-46.

18. Powers SK : Fenestration of intraventricular cysts using a flexible, steerable endoscope and the argon laser. Neurosurgery $18: 637-641,1986$

19. Rhoten RP, Luciano MG, Barnett GH : Computer-assisted endoscopy for neurosurgical procedures: technical note. Neurosurgery 40 : 632-638; discussion 638, 1997

20. Rhoton AL Jr, Gomez MR : Conversion of multilocular hydrocephalus to unilocular: case report. J Neurosurg $36: 348-350,1972$

21. Rohde $V$, Behm $T$, Ludwig $H$, Wachter $D$ : The role of neuronavigation in intracranial endoscopic procedures. Neurosurg Rev 35 : 351-358, 2012

22. Sandberg DI, McComb JG, Krieger MD : Craniotomy for fenestration of multiloculated hydrocephalus in pediatric patients. Neurosurgery 57(1 Suppl) : 100-106; discussion 100-106, 2005

23. Schultz $P$, Leeds NE : Intraventricular septations complicating neonatal meningitis. J Neurosurg 38 : 620-626, 1973

24. Schulz M, Bohner G, Knaus $H$, Haberl H, Thomale UW : Navigated endoscopic surgery for multiloculated hydrocephalus in children. J Neurosurg Pediatr 5 : 434-442, 2010

25. Teo C, Rahman S, Boop FA, Cherny B : Complications of endoscopic neurosurgery. Childs Nerv Syst 12 : 248-253; discussion 253, 1996

26. Zuccaro G, Ramos JG : Multiloculated hydrocephalus. Childs Nerv Syst 27 : 1609-1619, 2011 